\title{
Conocimiento Científico, CONOCIMIENTOS HETEROGÉNEOS Y CONSTRUCCIÓN DE PAZ: HACIA \\ UNA AGENDA DE INVESTIGACIÓN SOBRE POLÍTICAS Y GOBERNANZA DEL CONOCIMIENTO EN TRANSICIONES
}

\section{HACIA LA PAZ}

\author{
Alejandro Balanzó* \\ Carlos Mauricio Nupia** \\ Juan Pablo Centeno**** \\ Editores InVITAdos
}

\section{Resumen}

¿Cómo pueden ser más provechosas las relaciones entre fuentes heterogéneas de conocimiento -entre ellas el científico- para la construcción de paz? Tras un breve estado del arte para nutrir la discusión, el artículo aborda la pregunta construyendo sobre el concepto de diversidad epistémica. Se propone una heurística analítica comprensiva, fundamentada en ejercicios

\footnotetext{
* Doctor en Gobernanza del Conocimiento y la Innovación, Universidad de Twente (Países Bajos). Profesor titular, Universidad Externado de Colombia (Colombia). [alejandro.balanzo@uexternado.edu.co], [https://orcid.org/0000-0003-3002-5535].

** Doctor en Ciencia Política, Universidad Libre de Berlín (Alemania). Director Administrativo del Instituto Colombo-Alemán para la Paz (CAPAz) (Colombia). [carlos.nupia@instituto-capaz.org], [https://orcid.org/0000-0002-4758-239X].

**** Magíster en Gobierno y Políticas Públicas, Universidad Externado de Colombia. Investigador del Centro de Investigaciones y Proyectos Especiales (CIPE), Universidad Externado de Colombia (Colombia). [juan.centeno@uexternado.edu.co], [https://orcid.org/0000-0002-8068-4267].

Recibido: 14 de febrero de 2020 / Modificado: 16 de marzo de 2020 / Aceptado: 17 de marzo de 2020

Para citar este artículo

Balanzó, A., Nupia C. M., Centeno, J. P. (2020). Conocimiento científico, conocimientos heterogéneos y construcción de paz: hacia una agenda de investigación sobre políticas y gobernanza del conocimiento en transiciones hacia la paz. OPERA, $27,13-44$. DOI: https://doi.org/10.18601/16578651.n27.02
} 
académicos previos, que categoriza: 1$)$ niveles de gestión y aprovechamiento del conocimiento, 2) tipos y naturaleza del conocimiento en juego y 3) productos de conocimiento para la paz. Este texto describe y discute los artículos que hacen parte del presente dossier a la luz de tales referentes y sitúa algunos puntos de interés para una agenda de investigación: 1) expandir la reflexión sobre el conocimiento sobre o para la paz; 2) profundizar en los fenómenos de interacción entre la ciencia y otros tipos de conocimiento, lo que toca diversos repertorios, campos y niveles de interacción y modos de encuentro; y 3) retroalimentar el acervo teórico disponible en torno a la gobernanza de conocimientos heterogéneos en contextos transformativos, con especial atención a la teoría y prácticas de política pública en ciencia, tecnología e innovación.

Palabras clave: gobernanza del conocimiento; diversidad epistémica; política de innovación; construcción de paz; cogestión del conocimiento local; conocimientos heterogéneos.

\section{INTRODUCTION TO THE SPECIAL ISSUE: SCIENTIFIC KNOWLEDGE, HETEROGE- NEOUS KNOWLEDGE AND PEACE BUIL- DING: TOWARDS A RESEARCH AGENDA ON KNOWLEDGE GOVERNANCE AND POLICIES IN PEACE TRANSITIONS}

\section{Abstract}

How can relations between heterogeneous knowledge -including scientific knowledgebe more profitable to the aim of post-conflict peace? After a literature review, we tackle this question building on the concept of epistemic diversity. We propose a comprehensive analytical heuristic, grounded on previews academic encounters, using the following categories: i) knowledge profiting and management levels, ii) knowledge types at interplay, and iii) knowledge products for peace. We discuss the articles in this special issue in this light, finding some landmarks for a research agenda on the topic: i) expanding the scholarly reflection on knowledge for or about peace; ii) deepening the understanding of phenomena taking place in the interaction between science and other types of knowledge, specifically addressing the repertoires, fields, levels and encounter modes taking place, and iii) nurturing the theoretical base on governance of heterogeneous knowledges in transformative contexts, paying special attention to STI policy theory and practice.

Key words: Knowledge governance; epistemic diversity; innovation policy; peace building; local knowledge co-management; heterogeneous knowledge.

\section{INTRODUCCIÓN AL DOSSIER: UN EJERCICIO ACADÉMICO CONSTRUCTIVO}

Múltiples fuerzas plantean la pregunta sobre el papel y alcance de las políticas públicas de ciencia, tecnología e innovación (CTeI), así como de las organizaciones productoras de conocimiento (universidades, centros de investigación, colegios, organizaciones civiles, tanques de pensamiento, etc.) para el logro de transformaciones sociales. En el caso colombiano, el tema fue recurrente durante la negociación del Acuerdo Final para la Ter- 
minación del Conflicto y la Construcción de una Paz Estable y Duradera, firmado por el Gobierno y las Fuerzas Armadas Revolucionarias de Colombia-Ejército del Pueblo (FARC-eP) (Ordóńez-Matamoros, Centeno, Arond, Jaime y Arias, 2018), pero apareció también con la reciente iniciativa de política de ciencia e innovación para el desarrollo sostenible de Colciencias ${ }^{1}$, contenida en el denominado Libro Verde 2030: política nacional de ciencia e innovación para el desarrollo sostenible, que hace un llamado a adoptar un enfoque transformativo que contribuya a la solución de los grandes desafíos expresados en los Objetivos de Desarrollo Sostenible (ODS) de las Naciones Unidas. Estos esfuerzos denotan la intención de pensar en políticas y actividades contextualizadas, inclusivas y responsivas con respecto a las políticas tradicionales de CTeI, en un contexto que tiene la impronta de transición hacia la sociedad del conocimiento (Balanzó, Nupia y Centeno, 2019).

A pesar de lo anterior, las actuales políticas de CTeI soslayan y, eventualmente, desaprovechan las capacidades de los territorios en lo referente a la generación de conocimientos y saberes. Lo anterior cobra especial relevancia si se considera que la relación entre conocimientos provenientes de múltiples fuentes, construcción de paz desde las regiones y desarrollo humano y socioeconómico emergen como temas prioritarios en la agenda pública nacional del posacuerdo y en un contexto glo-

1 Hoy Ministerio de Ciencia, Tecnología e Innovación. bal en el que la relación entre ciencia y paz es cada vez más relevante ${ }^{2}$.

La pregunta acerca de cómo pueden ser más provechosas las relaciones entre distintas fuentes de conocimiento (no solo el científico) para la resolución de problemas emergentes en una sociedad en transición hacia la paz todavía está pendiente por responder. Más aún, en la literatura internacional sobre estudios de paz el tema es incipiente y es difícil encontrar revisiones sistemáticas y explícitas, vacío al cual se puede contribuir con análisis académicos hechos desde el caso colombiano (Pérez-Viramontes y Restrepo-Mesa, 2014; Hidalgo, 2014; Ordóńez-Matamoros et al., 2018; Rodríguez-Camargo y Ochoa-Duarte, 2018; Facio, 2019; Balanzó, Nupia y Centeno, 2019).

Frente a este panorama, el propósito del presente artículo es proveer insumos conceptuales y analíticos que permitan un mejor entendimiento sobre los retos de la gobernanza del conocimiento y la construcción de paz, pasando, naturalmente, por los que surgen para las políticas públicas en CTeI. El artículo también capitaliza ejercicios analíticos y reflexiones originales de los autores seleccionados para este dossier, en una perspectiva académica constructiva, y a la vez discute y describe sus trabajos.

El dossier nace de un esfuerzo sostenido que inició con las discusiones y los resultados del taller "Hacia una agenda de investigación sobre conocimiento científico, sociedad y construcción de paz en Colombia”, organi-

2 Ver Asamblea General de las Naciones Unidas (1988). 
zado conjuntamente por el Centro de Investigaciones y Proyectos Especiales (CIPE) de la Universidad Externado de Colombia y el Instituto Colombo Alemán para la Paz (CAPAZ) en diciembre de $2018^{3}$. El taller tuvo por objeto identificar problemas, enfoques, temas relevantes y objetos empíricos de investigación, en marcha o por desarrollar, que permitieran profundizar la comprensión de la relación entre producción de conocimiento científico, sociedad y construcción de paz. A este taller se postularon alrededor de 75 trabajos de investigación de los cuales fueron seleccionados 16 . Es importante resaltar que los trabajos seleccionados representan instituciones que trabajan en la producción de conocimiento sobre la paz, procedentes de distintas regiones del país.

De igual forma, el presente dossier se nutrió de la línea temática sobre "Ciencia, paz e inclusión social”, convocada en el marco del Congreso Internacional Gobernanza de la Ciencia y la Innovación. Hacia el Desarrollo Inclusivo, llevado a cabo en Bogotá entre julio y agosto de $2019^{4}$. Esta línea logró una amplia convocatoria a nivel nacional, esta temática fue la que recibió mayor número de postulaciones para el Congreso.

Dados estos antecedentes, los autores del presente artículo consideran que el tema ha

3 Ver más en: https://www.uexternado.edu.co/finanzas-gobierno-y-relaciones-internacionales/taller-haciauna-agenda-de-investigacion-sobre-conocimiento-cientifico-sociedad-y-construccion-de-paz-en-colombia/

4 El Congreso fue una iniciativa de la Red de Gobernanza y Gestión del Conocimiento, la Ciencia, la Tecnología y la Innovación -RedGCTI, con el apoyo de varias organizaciones. Consulte las memorias del Congreso en Peñuela Herrera (2019). logrado una importante acogida entre distintos tipos de público, y que la publicación del dossier es un esfuerzo adicional por capitalizar los aportes académicos sobre el mismo. De esta manera, se visibilizan campos de investigación emergentes en torno a la gobernanza del conocimiento, los estudios de conflicto y paz y el diseño de políticas públicas de CTeI.

\section{Ciencia, saberes heterogéneos y política pública: una discusión en ciernes}

Las políticas de CTeI se han diseñado e implementado históricamente como un motor de progreso, desarrollo y bienestar, en donde la innovación es una fuerza que contribuye a un mundo mejor, sin considerar sus posibles implicaciones y externalidades negativas, como tampoco la incidencia del avance tecnológico en la profundización de problemáticas sociales y ambientales (Schot y Steinmueller, 2018). Es necesario plantear la necesidad de formular políticas de CTeI y promover arreglos de gobernanza del conocimiento que consideren lo anterior, con una orientación que responda a los grandes retos sociales, como es el caso de la construcción de una paz sostenible en Colombia (Ordóñez-Matamoros et al., 2018). Esta reflexión se deriva del potencial de la CTeI en pro del desarrollo inclusivo y sostenible, particularmente en el contexto de países en desarrollo (Dutrénit y Sutz, 2014; Kuhlmann y Ordóńez-Matamoros, 2017).

Abordar los desafíos planteados por la construcción de la paz en Colombia está estrechamente relacionado con un cambio en la forma de concebir el desarrollo, lo que implica pasar de un énfasis en los "recursos" a un énfasis 
en las "capacidades", en donde las brechas y los encuentros o desencuentros entre conocimientos heterogéneos juegan un papel central. En ese sentido, resulta pertinente profundizar en el papel del conocimiento desde la perspectiva de las políticas públicas en el proceso de construcción de paz, sobre todo si se considera que además de los recursos y la voluntad política, este es una condición necesaria para el diseño y la implementación de políticas públicas viables (Ordónez-Matamoros et al., 2013).

En este punto, vale la pena preguntarse: ¿cómo pueden ser más provechosas las relaciones entre distintas fuentes de conocimiento (no solo el científico) para la resolución de problemas emergentes en una sociedad en transición hacia la paz? Para responder a dicho interrogante podría decirse que existen al menos dos enfoques que contribuyen a la discusión: uno denominado "moderno" y otro denominado "decolonial". El primero, más generalizado en el campo de análisis de las políticas públicas de CTeI, concibe la producción de conocimiento científico como la base del desarrollo socioeconómico incluyente y un medio para lograr el bienestar de la sociedad. Esta visión considera que las acciones/aportes realizados desde la academia, y, en general, desde las instituciones de investigación, son fundamentales para resolver los problemas derivados de la violencia, y, por tanto, aquellos que pueden dar origen a un conflicto armado. En el enfoque decolonial, por su parte, distintas formas de conocimiento, especialmente aquellas provenientes de las organizaciones sociales y las comunidades locales y ancestrales, se consideran claves para la resolución de los problemas generados por la violencia. Estas formas de conocimiento son bastante pertinentes, en la medida en que nacen de las mismas comunidades que padecen los conflictos.

La primera perspectiva (moderna) se puede apreciar en la emergente literatura sobre el tema que, si bien no aborda de manera directa y sistemática el papel de la gobernanza del conocimiento en la construcción de paz, destaca algunas experiencias y reflexiones que sirven para analizar el caso colombiano. Esta perspectiva se revisa en la primera sección del presente artículo. La segunda perspectiva (decolonial) puede entenderse desde la noción de diversidad epistémica, la cual se presenta en la segunda sección del artículo como marco conceptual y analítico para aproximarse al fenómeno de la existencia de distintas fuentes de conocimiento y a su coexistencia o rivalidad, reconstruido en parte con las discusiones que tuvieron lugar en el taller "Hacia una agenda de investigación sobre conocimiento científico, sociedad y construcción de paz en Colombia" (2018), cuyos resultados aportan una base empírica significativa y permiten plantear un primer modelo heurístico para entender la relación entre conocimiento/conocimientos y construcción de paz. En la tercera sección, se presentan los artículos de investigación que componen el dossier temático de la revista y se destacan los aportes principales de los mismos a la discusión sobre la gobernanza de la paz. A la luz del marco analítico presentado, lo descrito en las anteriores secciones sirve de base empírica para abrir una discusión sobre las posibles dinámicas que implica la gobernanza del conocimiento en escenarios de diversidad epistémica, lo cual se desarrolla en la cuarta sección. El artículo concluye con algunas 
reflexiones sobre posibles líneas futuras de investigación y el papel de la gobernanza del conocimiento en la transición hacia la paz. El objetivo es delimitar una agenda de investigación preliminar sobre el tema en Colombia.

\section{CONOCIMIENTO Y CONSTRUCCIÓN DE PAZ: UN CUERPO INCIPIENTE DE LITERATURA}

En una revisión sobre la evolución de los estudios de conflicto y paz, Alger (2007) reconoce que la investigación en este campo se ha concentrado en entender las causas de la guerra $\mathrm{y}$ de otras formas disruptivas que derivan en conflictos. Esto ha significado un avance importante en el conocimiento sobre medidas preventivas y estrategias para la construcción de paz a largo plazo. Sin embargo, Alger también resalta la dificultad de dar una identidad disciplinaria a un tipo de investigación que, como ocurre en el campo de los estudios de paz, casi siempre transcurre entre las fronteras disciplinarias. De igual forma, el autor destaca que el crecimiento de los estudios de paz ha tenido lugar en las instituciones de investigación y universidades, pero también se ha nutrido del conocimiento de organizaciones que afrontan constantemente conflictos sociales disruptivos (pp. 300-301).

Además del reconocimiento de la interdisciplinariedad de los estudios de conflicto y paz, Alger (2007) realiza un análisis de la emergencia de los instrumentos de paz diseñados en el marco del sistema de Naciones Unidas (p. 302). Este panorama de instrumentos de política está clasificado según los conceptos de paz negativa (eliminación directa de la violencia) o paz positiva (eliminación de las limitantes al potencial humano debido a las estructuras económicas y políticas). Una revisión rápida permite apreciar que instrumentos como el desarme y el control de armas son de los más antiguos (aparecieron en 1919), y que otros, como los derechos humanos, el desarrollo socioeconómico, la gobernanza de los bienes comunes, la educación para la paz, el balance ecológico o la perspectiva feminista fueron emergiendo a lo largo del tiempo y consolidando las temáticas del campo de investigación.

Si el debate sobre la naturaleza de los estudios de paz sigue abierto, debido precisamente a las distintas disciplinas involucradas, lo es aún más la reflexión sobre el papel del conocimiento científico o de otros tipos de conocimiento para entender el fenómeno de la construcción de paz. Aquí hemos logrado distinguir entre dos niveles de análisis: el conocimiento que se genera para investigar los temas de paz, que es principalmente el análisis de Alger (2007), y el conocimiento que se necesita para construir la paz como una opción viable, deseable y duradera, que es del que principalmente nos vamos a preocupar en este artículo.

La dificultad para diferenciar estos dos niveles de análisis se evidenció en la revisión de literatura sobre producción de conocimiento y construcción de paz que realizamos para el presente artículo. Utilizamos el Scimago Journal and Country Rank (SJR) para identificar un corpus de 22 journals, en el ámbito mundial y latinoamericano, relacionados específicamente con el campo de estudios de $\mathrm{paz}^{5}$. A partir de

5 Se utilizó la siguiente cadena de búsqueda a partir de las opciones ofrecidas por el sJR: socialsciences/political 
este corpus identificamos artículos publicados entre 2014 y 2019 que estuvieran relacionados con las siguientes temáticas: 1) relación entre conocimiento (uso y producción) y paz (construcción de paz o investigación en temas de paz y conflicto); 2) referencias al papel del conocimiento en el caso del proceso de paz en Colombia; 3) marco conceptual del campo académico denominado "estudios de paz" (peace studies). La idea detrás de esta búsqueda era la identificación de trabajos académicos que nos dieran pistas sobre cómo abordar la relación entre conocimiento y $\mathrm{paz}^{6}$.

Con base en esta revisión se puede concluir que el tema ha sido muy poco analizado. Los artículos que de alguna manera abordan la

science and international relations/all regions-countries. Posteriormente, se seleccionaron los journals cuyos nombres incluyeran en su título las palabras "paz" o "conflicto" y que estuvieran clasificados en las categorías Q1, Q2 y Q3, que son las de mayor impacto. De esta manera, identificamos un corpus de 12 journals en el ámbito internacional y 10 en el ámbito de América Latina: Journal of Conflict Resolution, Journal of Peace Research, Conflict Management and Peace Science, Cooperation and Conflict, Studies in Conflict and Terrorism, International Peacekeeping, Conflict, Security and Development, Peace and Conflict: Journal of Peace Psychology, Journal of Peace Education, Peace Economics, Peace Science and Public Policy, Journal of Peacebuilding and Development, Cultures et Conflits, Política y Gobierno, Revista de Economía Politica, Revista de Ciencia Política, Análisis Político, Izquierdas, Brazilian Journal of International Law, Revista Brasileira de Politica Internacional, Utopia y Praxis Latinoamericana, Colombia Internacional, Desarrollo y Sociedad.

6 Deseamos agradecer el apoyo de Sonja Smolenski y Anneke Siebeneck, practicantes del Programa ASA, quienes colaboraron en la identificación de artículos según los criterios definidos por los autores. Dicho trabajo fue realizado durante su tiempo de estadía en el Instituto Colombo-Alemán para la Paz (CAPAZ). producción de conocimiento están relacionados con temas como medioambiente, agenciamiento local, burocracia e instituciones, uso de tecnologías para la construcción de paz y conocimiento militar. Podría decirse que un punto común en sus enfoques es la construcción de una "paz positiva", es decir que sus análisis revisan el papel que juega el conocimiento para entender las causas que inhiben el potencial humano y, por ende, generan violencia, más que el de concebirlo como herramienta/medio para la finalización de la guerra.

En cuanto al análisis del caso colombiano, se encontraron referencias muy indirectas, relacionadas especialmente con el conocimiento experto que el sector militar ha ido acumulando. Esto es coherente con el avance de la literatura internacional, que ha estado particularmente interesada en explorar la relación entre innovación en el campo militar e innovación en el ámbito "civil". Estas son dos esferas que inicialmente contaban con una frontera claramente delimitada, pero que con el paso de los años se ha hecho más difusa (Molas-Gallart, 2010).

A continuación, enumeramos algunas entradas provistas por la literatura que sirven para abordar la relación conocimiento-paz:

- Construcción de paz y uso o explotación de recursos naturales. Temas como los conflictos derivados del cambio climático en la seguridad alimentaria, la gestión del agua y la minería ilegal hacen mención a la producción de conocimiento que se necesita para gestionar programas de intervención y construcción de paz. El concepto de "environmental peace ma- 
king" podría sintetizar la racionalidad de esta entrada (Reimers, 2016; Krampe, 2017; Ide, 2018; Marijnen y Schouten, 2019).

- Educación. El poder intrínseco de la educación y el conocimiento como factores de cambio es asociado a la promoción de la tolerancia, la inclusión social y el bienestar necesario en una etapa de posconflicto. La producción de pedagogías para la paz, el aprendizaje emocional y la producción de material educativo juegan un papel esencial en la producción de conocimiento (Diazgranados et al., 2014; Brynen, 2014; Eslami-Somea y Movassagh, 2014; Shehi, Ozcan y Hagen, 2018; Anctil y Bolívar, 2018).

- Agenciamiento y construcción de paz. Esta entrada se centra en el análisis de la potencialidad del conocimiento local para mediar en los conflictos y construir estrategias de apropiación comunitaria (local ownership). El capital social y las redes de conocimiento para la paz son algunos de los temas abordados en esta entrada (Yalçınkaya y Özer, 2017; Hetou,2018; Millar, 2018; Münch y Veit, 2018).

- Transferencia de conocimiento, burocracia $e$ instituciones. Esta entrada suministra evidencia sobre el papel que juegan las burocracias en la creación de instituciones de investigación para la paz, así como el poder de las instituciones internacionales como creadoras de conceptos y significados a través de la interacción entre "conocimiento experto" y "conocimiento local" sobre la paz (Lundgren, 2016; Bergmann, 2018; Oksamytna, 2018).
- Uso de tecnologías de la información y la comunicación (TIC) para la construcción de paz. Las TIC son un tema recurrente debido al papel que juegan en el manejo de los conflictos y en la construcción de un activismo para la paz. Análisis sobre la contribución de las TIC a la seguridad de las poblaciones afectadas por el conflicto o de dispositivos tecnológicos empleados en los sectores defensa o agrícola caracterizan esta entrada (Clubb, 2014; Baú, 2015; Spillane, 2015; Perera, 2017).

Con el fin de complementar la revisión de literatura, se decidió llevar a cabo una búsqueda más transversal que utilizara la totalidad de las palabras clave "paz" y "posconflicto" en combinación con nociones proxy de "conocimiento" (e.g. conocimientos, conocimientos locales, ciencia, tecnología, instituciones, desarrollo económico) ${ }^{7}$. Solo un artículo en la base de referencias muestra una discusión sistemática desde las áreas que usualmente abordan ciencia y tecnología (estudios sociales de ciencia y tecnología, sociología, economía, políticas públicas) y la paz: una propuesta investigativa alrededor de "innovación para la paz", desarrollada por Miklian y Hoelscher (2018), que plantea los desafíos conceptuales de este enfoque y discute formas de operacio-

7 Para el efecto, se hizo un ejercicio iterativo a través del motor de búsqueda integrado de la Universidad de Twente (google scholar, web of science, scopus, otras) y el motor de búsqueda LENs (lens.org). Los resultados arrojaron un número total de 169 ítems (U. Twente) y 85 ítems (Lens). La existencia de tal cantidad de artículos muestra que el conocimiento es un aspecto relevante y recurrente en la casuística alrededor de la paz. 
nalización del mismo. Los autores afirman que un mayor uso sistemático del conocimiento experto ofrece un potencial considerable para integrar la innovación tecnológica y la innovación social en la construcción de paz.

Para el caso colombiano, esta segunda búsqueda encontró que la literatura discute el potencial que tiene la CTeI para la construcción de paz en tres vías: 1) mediante la generación de oportunidades de desarrollo y de soluciones a necesidades estructurales, 2) como mecanismo para apoyar la implementación del Acuerdo de Paz de La Habana, y 3) como una aproximación al abordaje y solución de problemáticas territoriales (Facio, 2019). Un ejemplo de esto último pueden ser las iniciativas de innovación transformativa implementadas a nivel local y que tienen un potencial transformador en contextos de conflicto histórico a través del desarrollo y fomento de actividades productivas (Arond, Calvo, Plazas y Sotelo, 2018).

Por otro lado, interesa destacar el potencial que tienen los programas públicos de apropiación social del conocimiento, tales como el programa "Ondas", de Colciencias, para la construcción de paz por vía del fomento de una cultura de paz en niños, niñas y jóvenes (Pérez-Viramontes y Restrepo-Mesa, 2014). En ese mismo sentido, otros programas públicos como "Ideas para el cambio", "A ciencia cierta", "Programa jóvenes investigadores e innovadores por la paz" o "Programa de ciencia y tecnología para la paz en comunidades sostenibles" reflejan que existen aproximaciones sectoriales que contribuyen con inversión pública en conocimiento para la construcción de paz, por la vía de la innovación sostenible e inclusiva (Salazar, Lozano-Borda y LucioArias, 2014; Balanzó et al., 2019, p. 4).

En esa misma línea, cabe resaltar cómo desde lo público se destinan recursos de CTeI a través de mecanismos que implican una interacción entre distintos niveles de gobierno (nacional-territorial), como es el caso del Fondo de Ciencia, Tecnología e Innovación del Sistema General de Regalías (Hidalgo, 2014). Así, desde una comprensión amplia del concepto de paz positiva, existen diversos proyectos de inversión en CTeI que desde los departamentos de Colombia cuentan con potencial para contribuir a la construcción de paz por vía de, por ejemplo, fortalecer la "agricultura incluyente, equitativa y sostenible" y abordar factores que históricamente han contribuido a las dinámicas de conflicto y violencia en los territorios. Esto muy a pesar de que tal propósito no sea explícito para las inversiones de regalías en CTeI (Hidalgo, 2014, p. 43).

Otros trabajos ven en el contexto actual de construcción de paz, en general, y en el periodo posterior a la firma del Acuerdo de Paz de La Habana, en particular, un escenario de oportunidad para repensar el papel del conocimiento en términos de nuevas epistemologías y pedagogías (Rodríguez-Camargo y OchoaDuarte, 2018), así como de la política pública de CTeI hacia una orientación más responsiva (Ordóńez-Matamoros et al., 2018). Para que la política pública se oriente en ese sentido $\mathrm{y}$ aproveche las oportunidades del actual contexto, es necesario 1) que se fortalezca el entorno habilitador de la política pública, lo cual implica capitalizar experiencias existentes de CTeI que contribuyan a la construcción de paz; 2) que se fortalezca el entorno habilitador 
de saber científico pertinente, cuestionando y actualizando la institucionalidad de la investigación en Colombia; y 3) que exista un desarrollo temático diferenciado y multidisciplinar mediante la consolidación de una comunidad científica activa en lo referente al tema de la paz (Balanzó et al., 2019).

Las anteriores condiciones fueron planteadas por los autores de este artículo en un policy brief publicado por el Instituto CAPAZ, en el que se aborda el tema desde una racionalidad instrumental y de recomendación de política (Balanzó et al., 2019). En contraste, el presente dossier pretende avanzar en el abordaje académico de la temática, con el fin de plantear una agenda de investigación a largo plazo. En ese sentido, a continuación, se propone un marco conceptual útil para avanzar en dicha reflexión académica.

\section{HACIA LA CONSTRUCCIÓN DE UN MARCO ANALÍTICO PARA LA RELACIÓN CIENCIA-PAZ}

\section{Diversidad epistémica en la construcción de paz}

Esta sección pone la lupa sobre los retos y requerimientos para una mejor interacción de los conocimientos heterogéneos alrededor de la construcción de la paz. Para el efecto traemos a colación una discusión sobre la diversidad epistémica, que fuimos operacionalizando como marco analítico en las sucesivas rondas reflexivas cursadas por este proceso de construcción académica.

La diversidad epistémica se plantea como una reacción de la postura decolonial frente al patrón de poder colonial. Su motu es aquel de visibilizar, posicionar, visualizar, relacionarse con la diversidad a efectos de situar caminos alternativos. La noción de diversidad epistémica no tiene una definición concreta en la literatura decolonial. Opera, si se quiere, como un término sombrilla (Rip y Voß, 2013) que incluye un set variado de referencias y que se justifica en un set más bien heterogéneo de fuentes, referencias y justificaciones.

La diversidad epistémica aboga por una mirada más de fondo a la alteridad, y, con ella, una posición radical, en resistencia, que sitúa como punto de partida el reconocimiento del otro. Este punto de vista - o actitud quizás- se denomina transmodernidad según Dussel (1994), diversidad radical según Espinosa (2007) y pensamiento posabisal según Santos (2007). En tanto que perspectiva, la diversidad epistémica se efectúa de modos distintos.

La diversidad epistémica se manifiesta como supuesto, premisa, punto de partida. La noción ecología de saberes (Santos, 2006) denota la existencia de tal diversidad. Aquí el saber moderno/científico es uno más, y comparte con los otros un rasgo: la incompletud. Cabe aquí, sin embargo, recordar que el saber moderno/científico supone en sí mismo un estamento con prácticas institucionalizadas alrededor de ethos particulares en torno al cual tienen lugar prácticas sistemáticas de validación, y cuyos rasgos han sido estudiados en detalle siguiendo el aporte seminal de Merton (Orozco y Chavarro, 2010).

Ningún modo de saber es o podría ser completo, todos los modos de saber son parciales. Santos suma a la diversidad epistémica la ecología de temporalidades, que amplía aún 
más la perspectiva: lo contemporáneo expresa simultaneidad, pero no abarca las posibles direccionalidades en el tiempo (lineal, cíclico, circular, etc.) que plantean otras fuentes epistémicas, ni se agota en la lectura de un referente que opere como patrón único del tiempo presente.

Una segunda aproximación asocia la diversidad epistémica a un valor, al reconocimiento del otro en términos de su legitimidad intrínseca, a su encuentro y exploración desprevenida y desinteresada. Cajigas-Rotundo sugiere que "explorar en la práctica concreta [...] espacios liminares, trasegando umbrales, obliga a cuestionarse por lo que es y pueda llegar a ser el conocimiento en un mundo donde sean posibles la democracia y la diversidad epistémica" (2007, p. 190). Esta noción de democracia, asociada al reconocimiento inmediato del otro, recuerda aquella de Maturana (1994). En el trabajo de Santos (2007), aparece bajo la noción de ecología de los reconocimientos. Es aquello que permanece cuando se desecha la jerarquía, campo que exige la traducción como repertorio adecuado: la búsqueda de inteligibilidad sin negación o canibalización del saber distinto.

Una tercera aproximación asocia la diversidad epistémica a la creación de mundos sociales desde otras autonomías, bien sean de orden político o económico. Walsh (2007) explica una forma de interculturalidad que no se limita a "intercambio" o "folclorización", sino que más bien señala y significa procesos de construcción. Aquello que Walsh describe como la "construcción de un conocimiento otro, de una práctica política otra, de un poder social (y estatal) otro y de una sociedad otra; una forma otra de pensamiento relacionada con y contra la modernidad/colonialidad y un paradigma otro que es pensado a través de la praxis política”. Santos (2007) indica lo propio, en el campo económico, aludiendo a la ecología de las productividades.

Esta última lectura tiene varias implicaciones. En principio, se contrasta y amplía la metáfora decolonial, relativamente frecuente, según la cual otras epistemologías están fuera, son fronterizas (Mignolo, 2009), a una en la que esas epistemologías coproducen órdenes sociales. $\mathrm{O}$ dicho de otra forma, en la que el quehacer y el participar de esos otros también incide y deviene en la construcción social (Escobar, 2017; 2015; Ostrom, 1996). Aquí el trabajo de frontera (Gieryn, 1983; Mollinga, 2008) aparece como el proceso de demarcación en la construcción compartida de mundos sociales, no en su marginación. Esos "Estado otro", "práctica política otra", "sociedad otra" y "economía otra" coexisten, conviven y coconfiguran formas híbridas de Estado, política y sociedad. Si, por ejemplo, la resistencia deviene en políticas públicas, la configuración resultante mostrará modos de coexistencia de la diversidad, institucionalizada en modos híbridos de Estado.

En un contexto de globalización esta lectura dibuja un paisaje más complejo, distinto al colonial, pues expande el despojo también a las zonas "protegidas". O quizás permite la visibilidad de esos despojos: es diciente que en las últimas décadas la inequidad haya crecido dentro de y no entre países (Verbeek y Osorio, 2015). De Souza Santos observa que en este ámbito de creación de mundos sociales la ecología de las transescalas llama a la recupe- 
ración de universales ocultos o escondidos, y a su defensa a partir de la articulación translocal (García, 2014). Esto es, a la visibilidad, encuentro, comunicación y enredamiento entre actores que, sin ser homogéneos, estrategizan, demarcan, coconstruyen y coproducen en distintos campos sociales en torno a causas, identidades e intereses comunes.

Naturalmente, estos rasgos tienen efectos frente a la diversidad epistémica. Por un lado, visibilizan otras voces -otras formas de saber, locales, quizás menos distintas que las aborígenes- reivindicativas de saberes locales y críticas del régimen tecnocientífico (Cummings, Regeer, de Haan, Zweekhorst y Bunders, 2017). Por otro, y en términos de la ecología de las temporalidades, plantea otros posibles escenarios futuros de "gobernanza del conocimiento", en los que podrían coexistir, en paralelo, regímenes anclados en racionalidades diversas (van Oost, Kuhlmann, Ordóńez-Matamoros y Stegmaier, 2016).

La diversidad epistémica tiene límites como marco interpretativo. Si bien permite abrir un campo de indagación hacia otros saberes, y en general ayuda a visibilizar su ocultamiento, es un cuerpo de literatura que se queda corto en el planteamiento de conceptos operacionales que permitan profundizar la comprensión de su gobernanza. En este trabajo se intentará, de modo exploratorio y tentativo, que resultados del ejercicio académico cursado hasta la edición de este número ayuden a fundamentar algunas nociones. Esto es, llevar a cabo un ejercicio de pensamiento que intenta operacionalizar la comprensión del fenómeno de cara a su gobernanza y para ello, eventualmente, involucrar otras fuentes.
Tomamos como punto de partida la aproximación de Walsh (2007), en torno a la cual la diversidad epistémica tiene que ver con procesos de construcción en diversidad. Para la noción de gobernanza recurrimos a la heurística de colibración (Dunsire, 1996), que refiere a una imagen de posibles ajustes en los actores y sus modos de interacción en torno a posibles equilibrios alternativos que producirían resultados más deseables para el conjunto.

En tanto que proceso social concreto, la paz sitúa un contexto específico en torno al cual estos fenómenos tienen lugar. Se hace necesario identificar, evidenciar y comprender las dinámicas, los impactos y las tensiones que tienen lugar en las relaciones entre conocimientos heterogéneos, las características que cobra según los actores involucrados, las disciplinas en cuestión, los campos sociales en juego y, más aún, los elementos según los cuales la incomprensión de la diversidad epistémica pone en riesgo la misma paz.

Si el objetivo de conseguir la paz pasa por pensar no solamente en la desaparición de la confrontación armada, sino en la reducción de brechas de oportunidades que generan violencia y conflicto, entonces la identificación de puntos de conexión entre la perspectiva de los conocimientos heterogéneos y la del conocimiento científico tradicional puede desempeñar un papel relevante. Esta reflexión, que surge aquí coyunturalmente en torno al proceso de paz colombiano, en realidad tiene repercusiones en el marco de transformaciones globales más amplias, tales como el reto de transición a la sostenibilidad expresado en los oDs. 


\section{Modelo heurístico para la comprensión de la relación ciencia-paz}

Así pues, para efectos de capitalizar los aprendizajes cursados, ofrecemos aquí un primer modelo heurístico resultante de las discusiones realizadas durante el proceso explicado en la introducción del presente artículo. Estas categorías se perfilaron inicialmente en el taller "Hacia una agenda de investigación sobre conocimiento científico, sociedad y construcción de paz en Colombia”, y surgieron a partir de tres preguntas claves que se discutieron con los participantes:

- ¿Qué tensiones se presentan en la interacción entre el conocimiento científico y otros tipos de conocimiento que inciden en el entendimiento y la construcción de la paz?

- ¿Cuáles son los productos tangibles en que se concreta o se manifiesta la expresión "ciencia y tecnología para la paz"?

- ¿Cuáles herramientas conceptuales, materiales o metodológicas facilitan el diálogo entre el conocimiento científico y otros tipos de conocimiento en la construcción de paz?

La lógica subyacente a estas preguntas se basa en las siguientes suposiciones que sustentan el modelo:

1. Es reconocido que cualquier forma de producción de conocimiento experimenta tensiones de distintos tipos durante el proceso de su creación. Identificar estas tensiones permite ubicar un fenómeno concreto y observable, como es el caso de la construcción de paz, en el marco de una competencia de intereses de varios actores. En nuestro modelo, estas tensiones caracterizan la gobernanza del conocimiento.

2. Las iniciativas académicas y de investigación en materia de paz han derivado en productos de distintos tipos, que pueden ser considerados como productos de la investigación sobre la paz. Debido a la interacción de varios tipos de conocimiento que intervienen en el estudio y análisis de la paz, teóricamente no debería existir una sola forma de "salida" o "resultado" de dichos productos, sino que debería observarse un abanico diverso de los mismos que se acerca a los estándares tradicionales de la producción científica o al reconocimiento propio de otras formas de conocimiento. Un producto que mezcle estos tipos de estándares tiene la posibilidad de existir.

3. Si existen productos que mezclan estándares propios del conocimiento científico y de otras formas de conocimiento es porque debería haber una conexión, un punto de encuentro entre los conceptos desde donde parte el análisis o las metodologías que se utilizan para producirlos. Estas conexiones permitirían hablar, entonces, de un diálogo entre conocimiento científico y otras formas de conocimiento en relación con el tema de la paz.

En la figura 1 se pueden apreciar los elementos generales que conforman este modelo heurístico que sirve de base para entender la 


\section{FIGURA 1. MODELO HEURÍSTICO GENERAL PARA ENTENDER LA GOBERNANZA DEL CONOCIMIENTO EN LA RELACIÓN CIENCIA-CONOCIMIENTO-PAZ}

Fuente: elaboración propia.

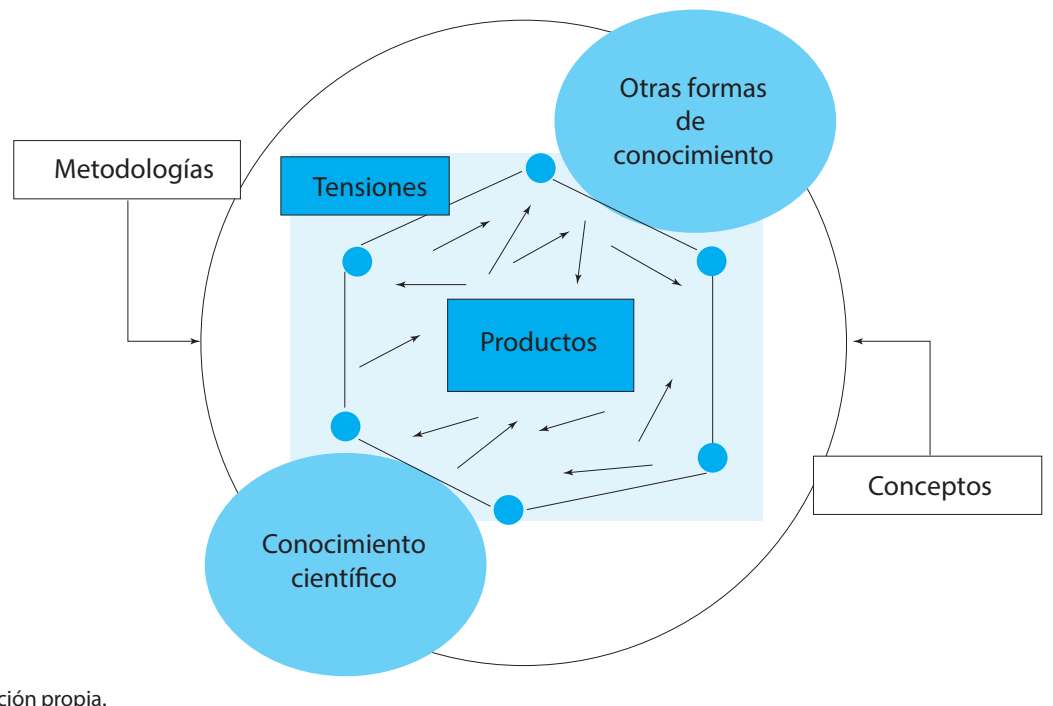

gobernanza del conocimiento en la relación entre ciencia, conocimiento y paz.

A partir de las discusiones realizadas en el taller mencionado, se pueden agregar las siguientes características a los elementos que conforman el modelo heurístico según los casos expuestos por los participantes:

- Las tensiones son manifestaciones propias de la gobernanza del conocimiento en relación con la construcción de paz y ocurren cuando: 1) se producen y generan conocimientos heterogéneos, 2) se usan los conocimientos heterogéneos, 3) se divulgan y apropian los conocimientos heterogéneos y 4) se confronta el conocimiento frente al poder político y se pone en escena en el marco de las políticas públicas. De manera general, estas ten- siones inciden unas sobre las otras y se manifiestan en dos tensiones adicionales: 5) las definiciones de objetos disciplinares, eventualmente, tanto como de agendas de investigación para el campo científico, y 6) las tensiones sectoriales/territoriales, evidenciadas en los usos y encuentros entre actores de raíces heterogéneas.

- Los productos se derivan del encuentro del conocimiento científico con otros campos sociales y otras formas de conocimiento. Se encontró que la naturaleza, el propósito, los alcances y las implicaciones de estos productos varían entre niveles (macro, meso y micro), pueden tener características diversas y plantean retos específicos. Es alrededor de la materialización de estos productos que tienen lugar las tensiones, $\mathrm{y}$ de donde surgen retos investigativos 
tanto como de gestión que aquí intentamos profundizar.

Este modelo heurístico plantea categorías relativamente simples, pero que integradas permiten una mirada más comprensiva sobre los retos de gobernanza -y allí de política pública- que enfrenta la gestión de conocimientos heterogéneos en torno al objetivo de la paz. La figura 2 ofrece un detalle de la ubicación de los productos de investigación sobre la paz, identificados en el taller, en relación con los niveles de gestión del conocimiento y los tipos de hibridación que se producen entre ellos. En ella se ilustra la diversidad de productos que aparecen como procesos requeridos para la cogestión local del conocimiento.

El valor de la cogestión local del conocimiento tiene que ver con su potencial práctico. De tal suerte que su realización no tiene lugar tanto en los extremos de los tipos de conocimiento (conocimiento local o conocimiento científico/experto $)^{8}$, sino en los distintos repertorios que se derivan de la interacción de estos extremos y que producen soluciones y modos híbridos.

8 En este punto vale la pena mencionar que en el taller se presentó una discusión sobre las particularidades del conocimiento cientifico en materia de paz y su diferencia con otros tipos de conocimiento. Por la naturaleza de los casos revisados, algunos de los cuales se exponen en los artículos que conforman este dossier, los autores decidieron utilizar los términos conocimiento local y conocimiento científico, para ubicarlos en los extremos de la figura 2 y así facilitar la clasificación de los productos. Esto se hace netamente por motivos heurísticos y no significa que haya existido un consenso en la elección de estos términos entre los participantes del taller.
En este contexto, aparecen como críticos los procesos de mediación y traducción de conocimientos. Estos tienen lugar en distintos campos y, tal como se muestra en la figura 2, dependen de múltiples enfoques y decisiones. También dependen de los diseños metodológicos de las investigaciones adelantadas por los distintos actores, de los enfoques sectoriales o intersectoriales de política pública, tanto como del direccionamiento de programas de investigación y desarrollo promovidos por instituciones y estamentos productores de conocimiento.

A continuación, se explican los ejes de análisis y la naturaleza de los productos identificados en el taller, a partir de los cuales se elaboró la figura 2

\section{Niveles de gestión y aprovechamiento del conocimiento}

Planteamos una categoría de niveles de gestión de conocimiento para describir el alcance social del que pueden participar la existencia, interacción y desarrollo de conocimientos heterogéneos. La noción de alcance social se refiere aquí a los órdenes y estamentos sociales en escena, en y entre los que pueden haber procesos de encuentro, interacción y generación a partir de conocimientos heterogéneos en torno al objetivo de lograr la paz.

El nivel macro describe un universo institucional o habilitante (que puede ser de política o relaciones entre actores de alcance global). El meso describe el nivel de las organizaciones y el encuentro entre estas. El nivel micro describe el grado de encuentro entre actores individuales. 


\section{FIGURA 2. PRODUCTOS DE INVESTIGACIÓN SEGÚN NIVELES DE GESTIÓN} Y TIPOS DE CONOCIMIENTO

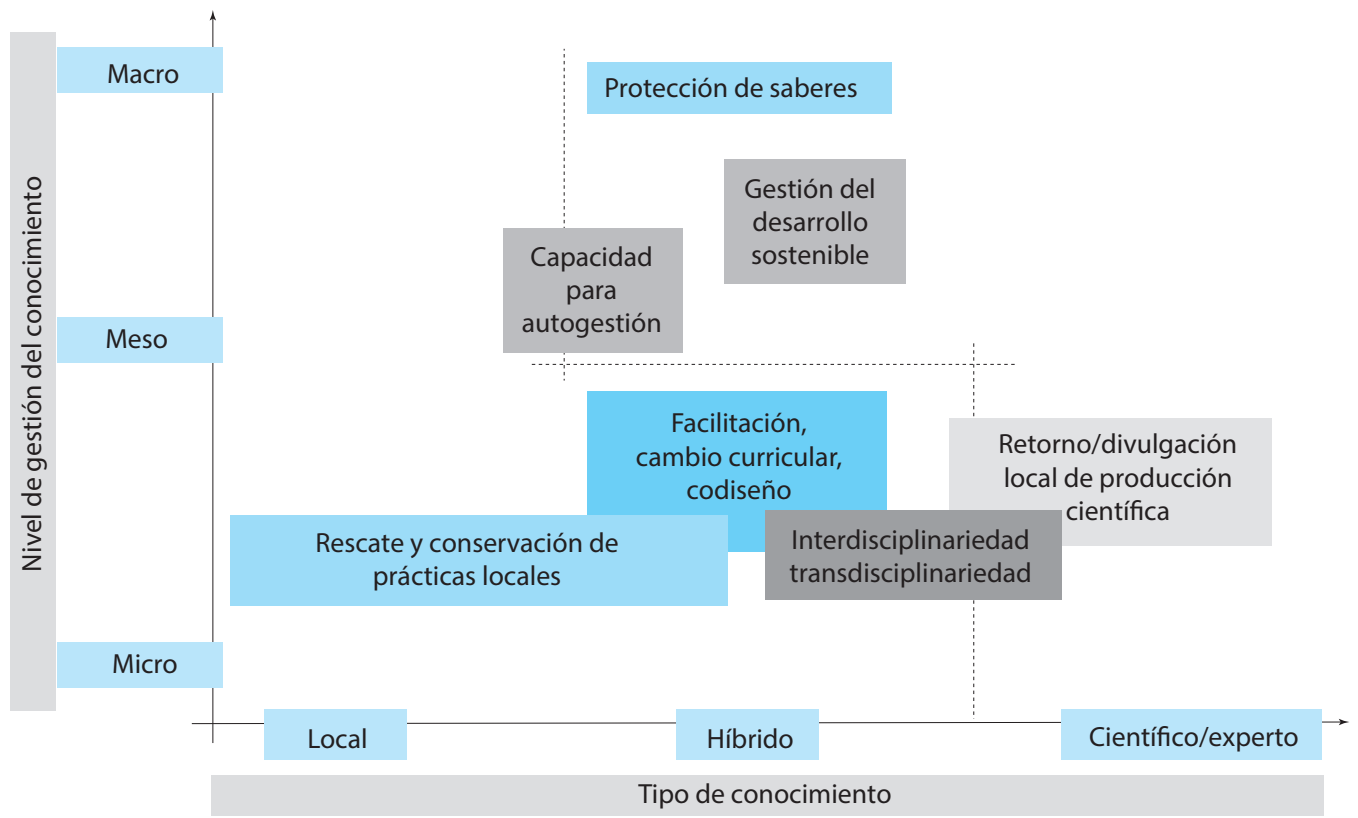

Fuente: elaboración propia.

\section{Tipos y naturaleza del conocimiento en juego}

Planteamos una noción muy genérica: tipos de conocimiento, para hacer referencia a la naturaleza de los conocimientos heterogéneos que interactúan en procesos de trabajo por la paz, y que sirve para realizar una lectura de flujos de conocimiento como insumo o producto de tales interacciones ${ }^{9}$. De modo muy

9 El problema de denominación y demarcación de otros tipos de conocimiento no es menor. En general, se utilizan expresiones como conocimiento local o conocimiento ancestral. Estas categorías son limitadas para abarcar la complejidad del conocimiento como fenómeno social, y en el marco de controversias actuales, son con frecuencia demeritadas frente a la ciencia, en lecturas más bien superficiales. Se ha preferido aquí general, se plantean tres tipos de naturaleza de los conocimientos:

1. El conocimiento científico o experto describe el universo de saberes producto del quehacer científico moderno y que pueden asociarse a sus principales instituciones y estamentos sociales (universidades y centros de investigación).

2. El conocimiento híbrido describe el universo en el que coexisten conocimientos heterogéneos de modos diversos. Esta coexistencia de saberes configura identi-

la noción de conocimientos heterogéneos para plantear una alternativa con otros posibles alcances. 
dades, productos sociales o estamentales, tanto como artefactos.

3. El conocimiento local describe el universo de saberes que tienen lugar en universos locales y que pueden o no derivarse de procesos indígenas o ancestrales. En todo caso, aquellos saberes que configuran en su expresión universos locales que operan más bien de modo adyacente a las instituciones y los estamentos sociales modernos.

\section{Productos de conocimiento para la construcción de paz}

Los productos identificados se pueden clasificar en tres grandes bloques: politicas, cogestión del conocimiento local y diseños apropiados.

Las politicas se entienden aquí como: 1) insumos discursivos y técnicos que plantean directrices para la acción; 2) herramientas e información que ayudan a la toma de decisión; o 3) insumos técnicos que sirven para proteger y aprovechar activos locales (tales como saberes o potencial aprovechable). En tanto productos, las políticas no solo se refieren a políticas públicas estatales en el sentido estricto, sino a aquellas líneas estratégicas de empresas, sectores, organizaciones de la sociedad civil, gremios, $y$, en general, cualquier actor social que está involucrado con la construcción de paz.

Como se ha planteado ya en otras reflexiones (Balanzó et al., 2019), algunos ejemplos de productos de política son:

- Políticas orientadas a pensar la gestión del conocimiento, la ciencia y la tecnología en órdenes locales. Interesan aquellas que incrementen la autonomía local; aquí aparecen, por ejemplo, mecanismos de apoyo a la descentralización. En algunos niveles estos procesos implican políticas que permiten la mediación (facilitación y divulgación), y traducción entre saberes locales y científicos.

- Políticas orientadas a rescatar y popularizar prácticas locales/ancestrales, y proteger conocimientos locales con mecanismos de patente colectiva.

- Sistemas de información que apoyan la toma de decisiones y que están liderados por gremios u organizaciones no gubernamentales, como es el caso de observatorios de derechos humanos, bases de datos que reposan en las cámaras de comercio o información productiva sectorial.

El segundo tipo de producto, la cogestión del conocimiento local, expresa la necesidad de consolidar infraestructuras locales de conocimiento. Discutida inicialmente en Balanzó et al. (2019), la cogestión del conocimiento local se efectúa con la valorización, circulación y apropiación de conocimientos en el marco del encuentro e interacción de fuentes epistémicas diversas (por ejemplo, saberes científicos, ancestrales, sectoriales, locales) que se expresan en productos muy concretos.

Naturalmente, estos procesos de cogestión exigen un ejercicio en torno a las capacidades de apropiación de conocimientos. Implican no solo abordar la dificultad implícita en propiciar el diálogo entre conocimientos, sino entender, comunicar y ubicar el lugar que en tal proceso ocupa el conocimiento científico. 
El tercer producto hace referencia a los diseños apropiados, es decir aquellos desarrollos (tecnológicos, metodológicos, técnicos o sectoriales) que tienen por propósito solucionar una necesidad inmediata en materia de investigación sobre la paz. La noción de diseños apropiados implica que estos desarrollos deberían ser absorbidos y gestionados de modo autónomo y sostenible por los actores y las comunidades locales (Balanzó et al., 2019). La figura 3 sintetiza ejemplos de productos de investigación apropiables por actores locales.

Llama la atención la diversidad de aproximaciones al tema de la paz que sugieren estos diseños. Algunos lo abordan tomando como referencia un campo problemático inmediato (lo ciudadano/político, lo relacionado con memoria, el problema de la justicia), mientras que otros productos lo abordan considerando el carácter habilitante o facilitador para la ocurrencia del conocimiento (lo productivo, lo público local).

En el desarrollo de estos diseños apropiados entran en juego tres aspectos básicos relacionados con las organizaciones productoras de conocimiento: 1) los métodos que se usan en la investigación, 2) los conceptos que se utilizan para construir su objeto de estudio, y 3) el lenguaje que utilizan para socializar su trabajo (Balanzó et al., 2019).

En materia de métodos, se identificaron tres grupos. El primero podría denominarse "formatos tradicionalmente académicos", en el que se registran actividades como seminarios con expertos e investigadores sobre conflicto y paz, talleres con comunidades, diplomados

FIGURA 3. DISEÑOS APROPIABLES POR ACTORES Y COMUNIDADES LOCALES

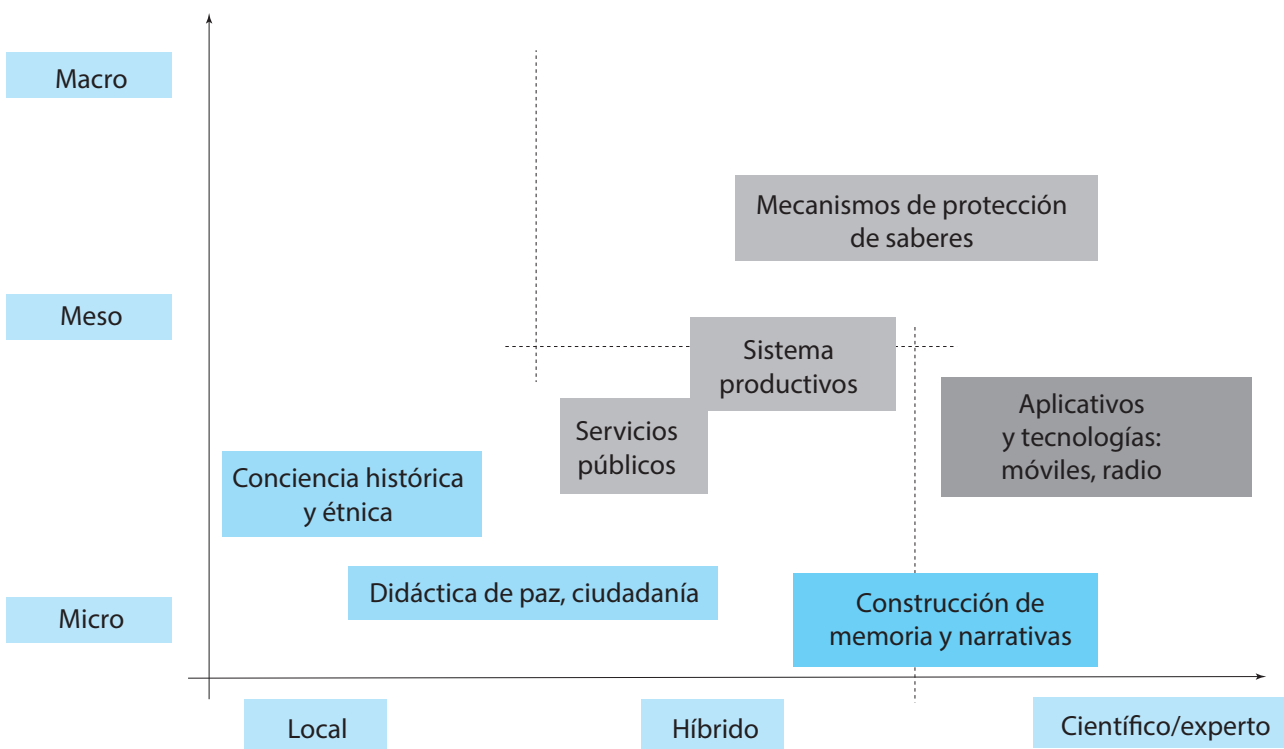

Fuente: elaboración propia. 
de capacitación a actores involucrados en el conflicto o servicios legales ofrecidos por los consultorios jurídicos (para el caso específico de una disciplina como el derecho).

El segundo grupo de métodos podría denominarse "métodos participativos". En este grupo se incluyen herramientas como el diagnóstico local participativo, la construcción de cartografías sociales, la permanencia de investigadores en los territorios afectados por la violencia durante espacios largos de tiempo con el fin de "habitarlos" y entenderlos, o la construcción de planes territoriales que mencionan específicamente al conocimiento como fuente de desarrollo (tales como los planes de desarrollo con enfoque territorial - PDET).

Finalmente, el tercer grupo podría denominarse "métodos mixtos", y se refiere a casos como el "aprendizaje basado en proyectos", que reúne herramientas tradicionalmente utilizadas en la academia (el diseńo del proyecto de investigación) con metodologías de corte más participativo utilizadas por actores sociales para la resolución de problemas y conflictos locales. También guardan relación con este grupo de métodos las Tic, que se identifican como interfaces para recolectar y sistematizar información primaria para la producción de conocimiento científico, pero al mismo tiempo se ven como herramientas que pueden resolver problemas concretos como la comercialización de productos agrícolas, el monitoreo de las condiciones climáticas que afectan un cultivo o la protección de poblaciones en riesgo de ser afectadas por la violencia de un conflicto.

En materia de los conceptos que sirven para articular los distintos tipos de conocimiento involucrados en la investigación sobre la paz, la cocreación y el codiseño merecen un lugar especial por lo constante de su utilización. La cocreación hace referencia a un proceso colectivo de creación que involucra múltiples partes y escalas, por tanto, ofrece una perspectiva más horizontal de la producción del conocimiento. El codiseńo, por su parte, es un concepto que sirve para superar la tensión entre conocimiento científico y no científico. El codiseño parte de la idea de que existe un conocimiento difuso (todas las personas tienen la capacidad de diseñar algo) que potencia el trabajo en el territorio.

Bajo esta sombrilla de la cocreación aparecen también conceptos de corte más disciplinar como es el caso de la "biomémesis" (observación de la naturaleza como guía, mentor y modelo de acción); el modelo osce (Open Source Circular Economy) que genera estándares para que distintas comunidades trabajen con énfasis en la sostenibilidad ambiental; y los conceptos de epistemologías plurales y cosmolocalización que se refieren a la idea de que el conocimiento es situado y depende del territorio en el que se desarrolle.

Por último, nos referiremos a la categoría del lenguaje, la cual incide en la apropiación de los productos de investigación. Esta es una categoría amplia y compleja que necesita ser refinada. Se refiere particularmente a la necesidad de construir palabras y discursos que eviten la exclusión de "el otro" y reconozcan el poder "transformativo" que tiene el lenguaje. El uso de un lenguaje incluyente facilita acceder/entender/reconocer otras formas de conocimiento. 
PRESENTACIÓN DEL DOSSIER: APORTES A LA REFLEXIÓN SOBRE EL ROL DEL CONOCIMIENTO Y LA CTEI EN LA TRANSICIÓN HACIA PAZ

Los autores de los artículos de este número especial de la revista Opera realizan aportes relevantes a la discusión presentada anteriormente, brindando evidencia empírica sobre prácticas locales de gobernanza del conocimiento, procesos de generación de conocimiento sobre y para la paz, e intersecciones entre ciencia y paz. En ese sentido, actualizan la reflexión sobre cómo las políticas públicas y la gobernanza del conocimiento y la CTeI dialogan con los procesos de construcción y transición hacia la paz a nivel territorial. Aunque estos trabajos no siguen como referente el modelo heurístico antes descrito, pues su propósito fue precisamente analizar casos desde diversas perspectivas para poder dar una mirada más espontánea al estado de la discusión, sirven para discutirlo e interpelarlo.

En efecto, los casos documentados en este dossier ilustran un abanico variado de entradas y aproximaciones, que permitirán en lo que sigue una discusión más nutrida. Quinchoa, en su artículo "Aportes a la apropiación social de TIC desde consejos comunitarios afrocolombianos de Corinto, Cauca”, expone la experiencia de apropiación social de las TIC en dos consejos comunitarios afrocolombianos del departamento del Cauca, abordando las motivaciones de dichas iniciativas y sus implicaciones en las organizaciones rurales afrocolombianas. El autor propone una estrategia metodológica de apropiación social de TIC y facilita la intersección entre prácticas locales de conocimien- to heterogéneo, ejercidas desde los consejos comunitarios y dispositivos de conocimiento tecnocientífico involucrados en el uso y apropiación de las Tic.

Cisneros, Pemberthy y Chaguendo, en el artículo "Gestión del conocimiento: una apuesta desde la gobernanza para la educación en salud en el Cauca”, presentan un estado del arte, sistematización y caracterización de las dinámicas de apropiación social en procesos de información, educación y comunicación a partir del caso de la educación en salud, también en el departamento del Cauca. Las autoras ven en la apropiación social un mecanismo de gobernanza útil para facilitar mayor participación comunitaria y, por esta vía, aportar a la reconstrucción del tejido social que contribuya a una sociedad más equitativa y en paz.

Bedoya-Dorado, Castro-Peńa y HoyosBravo analizan la articulación entre los emprendimientos rurales y las apuestas de construcción de paz en el Valle del Cauca, desde las nociones de ecosistema de emprendimiento y de emprendimiento rural. En su artículo "El emprendimiento rural en la construcción de paz: análisis de la (des)articulación en el Valle del Cauca, Colombia”, los autores exploran la relación entre los negocios emprendedores y la construcción de paz, asunto de especial relevancia si se considera que la innovación emprendedora tiene el potencial de aportar a la construcción de paz en escenarios en donde las pocas oportunidades han derivado en repertorios de conflicto (Miklian y Hoelscher, 2018).

Cárdenas, Tocarruncho y Lerma, en el artículo "Tendencias de la investigación sobre reintegración y reincorporación de excomba- 
tientes en Colombia. Tensiones y oportunidades", identifican patrones, tendencias, actores, temas recurrentes y metodologías empleadas en investigaciones de la comunidad científica para la generación de conocimiento sobre reintegración y reincorporación de excombatientes en Colombia, apoyadas por la Agencia para la Reincorporación y la Normalización (ARN). Con esto, los autores aportan a la comprensión de las lógicas de producción de conocimiento sobre la paz, que a la larga resultan útiles para la gobernanza de los procesos de reintegración y reincorporación en este contexto.

En contraste, y con una vocación mucho más orientada hacia el conocimiento para la paz, en el artículo "Ingeniería para la construcción de paz: una reflexión preliminar para procesos tecnocientíficos de resiliencia territorial", Reina Rozo propone el enfoque de la Ingeniería para la Construcción de Paz (IcP) como una praxis alternativa del ejercicio de esta disciplina en escenarios de conflicto, posconflicto y en situaciones donde la tecnociencia afecta especialmente a comunidades marginadas y excluidas, lo que genera aún más inequidad. El autor discute sobre las responsabilidades y oportunidades de la ingeniería en la paz y la resolución de conflictos, en donde la participación activa de diversos actores en las prácticas ingenieriles y de CTeI pueden contribuir a la superación de la violencia.

Ángel Botero, en su artículo "Ciencia y paz en interferencia", reflexiona sobre las interferencias entre ciencia y paz a partir del seguimiento y estudio de cómo los biólogos realizan los inventarios de especies durante las expediciones del programa "Colombia Bio" financiado por Colciencias. De esta manera, la autora aporta a la comprensión de cómo la justicia transicional se relaciona con prácticas científicas como la de inventariar la naturaleza. La autora explora cómo en este contexto la paz facilita y amplía la práctica científica y cómo la paz tiene una incidencia profunda en la significación de los objetos científicos, concretamente los biológicos, con el descubrimiento de las "especies de la paz".

Finalmente, en la sección de tema libre, En el texto "Cambio climático en la región Noreste del Uruguay: clivajes en las percepciones de los actores territoriales", Stuhldreher analiza la percepción de los actores territoriales acerca de los impactos del cambio climático en la región Noreste de Uruguay, así como sus visiones acerca de las políticas y los mecanismos de la gobernanza climática, tras diez años de la creación del Sistema Nacional de Respuesta al Cambio Climático (sNRCC) en ese país. Por su parte, en el artículo "Entre el petróleo y la democracia: el mecanismo causal que une la liberalización petrolera y el fortalecimiento de controles democráticos en la política petrolera colombiana (2002-2018)", Ruiz explora la relación causal entre liberalización de la industria petrolera y el fortalecimiento de los controles democráticos a partir del caso colombiano, por vía de una implementación participativa y descentralizada de las agencias estatales de petróleo.

\section{DISCUSIÓN: GOBERNANZA TRANSFORMATIVA EN AMBIENTES EPISTÉMICAMENTE DIVERSOS}

Si bien cada uno de los artículos que componen el número especial realizan contribuciones 
empíricas y conceptuales diversas y particulares a la comprensión de la gobernanza del conocimiento en la transición hacia la paz, a continuación nos interesa explorar lo que estos en su conjunto pueden decirnos sobre la pregunta central del presente artículo: ¿cómo pueden ser más provechosas las relaciones entre fuentes heterogéneas de conocimiento para la resolución de problemas emergentes en una sociedad en transición hacia la paz?

Para esto, discutimos y sintetizamos las ideas y los aportes de los artículos en clave de las tres categorías transversales propuestas en la figura 2 que profundiza el modelo heurístico anteriormente propuesto mediante la ubicación de los productos de investigación para la construcción de paz en relación con los niveles de gestión del conocimiento y los tipos y la naturaleza del conocimiento en juego.

Interesa comenzar llamando la atención sobre un límite que presenta este ejercicio: los trabajos no son necesaria o suficientemente diversos epistémicamente. Tiende a haber más reflexiones desde el lugar ciencia hacia otros lugares. Y como tal se presume a los otros en interacción. Esto no resta legitimidad a las reflexiones como tal, pero sí devela tareas pendientes de validación y profundización del ejercicio en múltiples direcciones, en torno a los fenómenos que tienen lugar en el encuentro entre conocimientos heterogéneos, en términos tanto de flujo como de contenido. El demarcamiento de fronteras propio de la práctica científica (Gieryn, 1983) impide una lectura más espontánea de aquellos otros con los que interactúa. Este tomo constituye un ejemplo de este límite.

\section{Niveles de gestión y aprovechamiento del conocimiento}

La coconstrucción surge en múltiples niveles. Son frecuentes en estos trabajos los niveles meso y micro. En el nivel micro, los contextos rurales, comunitarios y territoriales resaltan como espacios de experimentación que sirven como lugar de encuentro entre actores científicos y no científicos, en los que se gestan prácticas de conocimiento alternativas para la construcción de paz (Reina), se facilitan y profundizan actividades de apropiación social del conocimiento (Quinchoa), y se adelantan iniciativas de emprendimiento desde una lógica de abajo hacia arriba (bottom-up) (BedoyaDorado et al.).

Estos trabajos presentan iniciativas valiosas con alcances limitados frente a su potencial. Se sitúan en entornos de práctica usualmente ajenos a la preocupación de políticas sectoriales CTeI, pero claramente están sujetos a las condiciones de los espacios meso y macro que les habilitan. En clave de la perspectiva multinivel de cambio sociotécnico (Geels, 2002), el régimen condiciona la naturaleza y los alcances del relacionamiento de frontera que tiene lugar entre conocimientos heterogéneos (Gieryn, 1983). En la literatura de políticas transformativas de CTI, se esperaría que estos nichos -i.e. espacios protegidos para la experimentación- sean la base para el escalamiento de innovaciones (Schot y Geels, 2008).

Queda por profundizar el papel que en los entornos en los que tienen lugar estos trabajos juegan 1) los regímenes como objeto y lugar de tensión, coexistencia o transformación (incluyendo el sector CTI, pero también otros 
estamentos); 2) el alcance, los retos y costos de cogestión local de organizaciones y regímenes configurados sobre conocimientos otros que no comparten el universo epistémico moderno; y 3) el alcance y la racionalidad de actores y estamentos habilitadores de nivel meso y macro de cara a la posibilidad de la profundización y el escalamiento de tales iniciativas.

En efecto, la transformación de racionalidades de política pasa también por el trabajo conceptual y crítico que permita dar soporte a los enfoques transformativos (e. g. Grin, Rotmans y Schot, 2010; Schot y Steinmueller, 2016; 2018) a fin de superar sus propios límites. Esto incluiría, por ejemplo, en el caso de Reina, efectos en política de discusiones de índole disciplinar alrededor del diseño.

Por su parte, algunos trabajos llaman la atención sobre alcances y retos en la articulación entre los niveles macro y micro, concretamente en el diálogo y el lugar de los actores locales en la política pública, tal como se discute en artículos sobre la validación experta en procesos de justicia y fenómenos discursivos que configuran la relación entre ciencia y paz (Angel; Bedoya-Dorado et al.), o a la mediación de los gobiernos locales en torno a prioridades sectoriales (Cisneros et al.).

\section{Tipos y naturaleza de conocimiento en juego}

En principio, interesa notar que la coconstrucción entre saberes muestra distintos modos de aprovechamiento híbrido. La figura 2, "Productos de investigación según niveles de gestión y tipos de conocimiento", muestra al menos dos repertorios de relacionamiento entre fuentes de conocimiento aparte de la oferta científica. En el primero, el conocimiento local nutre o alimenta un producto de conocimiento posible (e. g. saberes sostenibles y conocimientos ancestrales), en lo que configura una forma de aprovechamiento.

En el segundo, la interacción entre saberes locales y saberes científicos fortalece capacidades (e. g. políticas y autogestión), en lo que configura una forma de colaboración ${ }^{10}$. Esta última supone (y requiere) legitimidad recíproca. Por ejemplo, aunque en muchos casos con retos de coordinación, el conocimiento local se apoya en las iniciativas de emprendimiento rural, un posible vehículo que lo proyecta a puntos de encuentro con iniciativas de paz del nivel de política, diseñadas desde una racionalidad tecnocrática (Bedoya-Dorado et al.). En otros casos, es el mismo investigador el llamado a facilitar y mediar la interacción entre saberes locales y saberes científicos para el fortalecimiento de capacidades por vía de procesos de, por ejemplo, apropiación social del conocimiento y la tecnología (Quinchoa), o del diálogo de saberes en donde el codiseño se destaca como una práctica central (Reina).

Esta construcción híbridase manifiestatambién en los encuentros entre campos de práctica distintos que convergen en objetos del entorno, como lo ilustra Ángel para el caso del proceso de inventariar especies en el contexto de construcción de paz. Aquí, la idea que subyace al concepto de justicia transicional y las

10 Hay un cuerpo de literatura abultado en lo que se refiere en particular al relacionamiento entre ciencia y políticas públicas, por ahora reducido al sector ciencia y tecnología: Colebatch, Hoppe y Noordegraaf (2010); Hoppe (2010b, 2005); Jasanoff (2004). 
ciencias naturales converge en la coconstrucción de objetos de conocimiento, como lo son nuevas especies inventariadas. La paz deja de ser, entonces, un fenómeno perteneciente estrictamente a un plano político y trasciende al plano científico, al tiempo que permea diversos niveles de interacción, de lo macro a lo micro.

Para todos los casos, la visión de éxito tiene que ver con la mejor hibridación posible entre fuentes diversas: poner en juego aquel saber que es requerido a fin de concretar un activo colectivo deseable. Esto supondría disposición y entornos habilitantes que permitan un relacionamiento de frontera adecuado a efectos transformativos (Balanzó, 2016).

\section{Productos de conocimiento para la construcción de paz}

Antes de discutir los productos de conocimiento objeto de este número, cabe notar que los trabajos exhiben diversidad en términos científicos disciplinares. Hay productos de las ciencias naturales (e. g. biología, ciencias agronómicas, ecología), así como productos de las ciencias sociales (e. g. antropología), y aplicadas (diseño, derecho, administración pública).

Es probable que cada disciplina y campo disciplinar tengan modos específicos de relacionamiento con los ámbitos sociales. Aquí hay un campo de interés investigativo alrededor de posibles ethos diferenciados. En ese sentido, un asunto recurrente en los artículos del dossier es el involucramiento del investigador con los contextos, las comunidades y los objetos estudiados: hay un foco en metodologías de nivel micro, frente al cual algunos artículos proponen enfoques de relacionamiento con contextos de diversidad epistémica (Reina; Quinchoa; Cisneros et al.).

Asimismo, hay diversidad en términos de los campos de práctica desde los cuales se expresan esos saberes locales. Los niveles de gestión macro y meso (e.g. campos de política y cogestión del conocimiento local) describen ámbitos de saber técnico-administrativo. En el nivel micro puede haber saberes técnicos (productivos) y administrativos (públicos o empresariales). Más importante aún: hay implícito un tipo de saber local fundamental referido a las lógicas organizacionales autónomas. El dossier aporta algunos elementos de referencia que ilustran esta discusión, entendiendo las organizaciones como fenómenos culturales profundamente influenciadas por el contexto en el que operan. Este es el caso de los consejos comunitarios afro, como estructuras operativas que se desarrollan según lógicas ortodoxas de la administración (Quinchoa).

Esta esfera de conocimiento tiene que ver con la sostenibilidad en el tiempo de los procesos de gestión de conocimiento, bien para su aprovechamiento inmediato (como producto), como mediato (como capacidad intrínseca). En ese sentido, algunos de los artículos del dossier informan sobre metodologías por seguir en procesos de gestión y apropiación social del conocimiento, que sirven como producto de aprovechamiento inmediato (Quinchoa; Cisneros et al.), y otros sobre casos exitosos de fortalecimiento de capacidades por vía de prácticas de codiseño, aspecto que contribuye a la construcción de paz desde la CTeI de aprovechamiento mediato (Reina). 


\section{A MANERA DE CONCLUSIÓN: HACIA UNA AGENDA DE INVESTIGACIÓN SOBRE CONOCIMIENTO, SOCIEDAD Y CONSTRUCCIÓN DE PAZ EN COLOMBIA}

Este dossier supone una primera aproximación a la pregunta acerca de cómo pueden ser más provechosas las relaciones entre distintas fuentes de conocimiento (no solo el científico) para la resolución de problemas emergentes en una sociedad en transición hacia la paz. Si bien este tiene un alcance aún exploratorio, orientado a notar posibles rutas de entrada a la pregunta, en este artículo se presentan a manera de modelo heurístico algunos elementos conceptuales y empíricos que aportan referentes tentativos para profundizar en la consolidación de una agenda de investigación sobre la relación entre ciencia, conocimiento y paz.

\section{Conocimiento sobre o para la paz}

El primer punto que se debe tener en cuenta en una agenda futura de investigación es la pregunta de si existe una diferencia entre la producción del conocimiento necesario para investigar el fenómeno de la paz (conocimiento sobre la paz) y la producción del conocimiento necesario para lograr o construir una paz sostenible (conocimiento para la paz). Tentativamente, se podría decir que la diferencia podría estar en el uso o no de un método avalado con los estándares académicos y científicos de las ciencias sociales. Sin embargo, las reflexiones de Alger (2007) sobre la conformación disciplinar del campo de estudios de paz y conflicto plantean al menos la duda sobre la pertinencia decircunscribirúnicamentea las metodologías de este tipo el estudio de uno de los grandes desafíos de la sociedad en el siglo XxI.

El modelo heurístico planteado en este artículo, y la revisión de literatura hecha sobre el tema, permiten avanzar en esta línea. Una caracterización más sólida de los productos de investigación relacionados con temas de paz y el entendimiento más sistemático de la forma en que se llega a ellos, podría arrojarnos luces sobre si el conocimiento para entender el fenómeno de la paz y el conocimiento necesario para evitar que se genere conflicto y violencia guardan similitudes.

Esto implica también entender mejor los ethos disciplinares y las particularidades de la práctica científica en torno al tema de la paz, de modo que puedan escalarse mejor sus productos e impactos, e indagar sobre los roles y las prácticas diversos que se desarrollan en el marco de múltiples disciplinas o subdisciplinas.

Un asunto que no escapa a esta discusión tiene que ver con la reflexión sobre la agenda de investigación misma y su eventual "gestión". Concretamente, inquieta el asunto de cómo se relacionaría esta con otras agendas de investigación. Algunas preguntas en ese sentido son:

- ¿Cuáles son sus modos específicos de relacionamiento con los "otros"?

- ¿Cómo dialoga con otras nociones para demarcar y encontrar puentes?

- ¿Qué temas requieren mayor sistematicidad? 


\section{Interacción entre conocimiento científico y otras formas de conocimiento}

Sin lugar a dudas, el modelo heurístico aquí presentado se basa en la conversación posible entre el conocimiento reconocido y validado como científico, y el conocimiento presuntamente producido con otras metodologías y formas de validación. Aquí la discusión es tan grande como el mismo problema planteado por la filosofía de la ciencia. Sin embargo, la aparición de una diversidad de productos de investigación relacionados con el campo de la paz ofrece un punto de partida importante para develar formas de conexión entre estos dos extremos del continuum, presentados en el modelo heurístico.

Existen todavía varios cabos sueltos cuando la diversidad epistémica aparece en el centro de la discusión. El primero de ellos tiene que ver con racionalidades dominantes en el entorno científico frente a la naturaleza y diversidad de sus propios productos. Para fines transformativos, es claro que la diversidad disciplinar, la interdisciplinariedad y la transdisciplinariedad aparecen como prácticas usuales en el quehacer científico, más connaturales a sus estándares de seguimiento.

El segundo de ellos aborda los extremos de la diversidad. Aquí los saberes otros aparecen tenues en diferencia y se intuye que podrían ser en efecto tratados como nichos. Una mirada no tan profunda permite ver que este no es el caso, y que se requiere aún de filigrana para entender cómo es que regímenes paralelos de saber se relacionan con el régimen tecnocientifico.

Los resultados aquí esbozados son optimistas y muestran escenarios deseables de aprovechamiento y colaboración entre saberes, por ejemplo. Es posible que alrededor de objetos que generen más controversia (e. g. semillas o hidrocarburos) los escenarios de encuentro en frontera sean distintos y marquen horizontes en los que saberes y ciencia están capturados en fenómenos de gobernanza global controversiales.

La discusión aquí realizada permite identificar necesidades de investigación en distintas vías:

- Esbozar mecanismos de medición de procesos de intermediación y traducción de saberes.

- Profundizar el conocimiento y la gestión en la relación entre disciplinas científicas, prácticas sectoriales y políticas públicas.

- Profundizar el conocimiento sobre repertorios y contenidos de conocimiento que tienen lugar en torno a la cogestión local del conocimiento, y lo que ello requiere en términos de acompasar racionalidades $\mathrm{y}$ ritmos entre actores.

- Profundizar el conocimiento sobre tipos y modos de hibridación de saberes que tienen lugar en estos procesos.

\section{Gobernanza del conocimiento}

Dado que la diversidad epistémica coconstruye en varios sectores y niveles de gestión sociotécnica mostrados en el modelo heurístico, su gobernanza supondría atención para tales niveles. La perspectiva multinivel que subyace a la racionalidad del enfoque de políticas transformativas ha probado ser una herramienta útil en entornos de transición (Elzen, Geels y Green, 
2004; Geels, 2002; 2010). Cabe preguntarse en qué medida es factible que pueda mostrar caminos para efectos de interacción entre saberes a nivel de régimen. En ese sentido, algunos de los interrogantes por explorar que emergen tienen que ver con:

1. Los regímenes como objeto y lugar de tensión, coexistencia o transformación (incluyendo el sector CTeI, pero también otros estamentos).

2. El alcance, los retos y costos de cogestión local de organizaciones y regímenes configurados sobre conocimientos otros que no comparten el universo epistémico moderno.

3. El alcance y la racionalidad de actores y estamentos habilitadores de niveles meso y macro de cara a la posibilidad de la profundización y el escalamiento de tales iniciativas.

\section{Racionalidad de las políticas públicas de ciencia, tecnología e innovación}

Es necesario documentar las dinámicas en las que el conocimiento local nutre o alimenta un producto de conocimiento que luego es aprovechado en la construcción de paz. Aquí, tanto la política pública (policy) como la política (politics) son objetos susceptibles de mayor indagación, ahondando en las relaciones de poder que atraviesan las prácticas y los encuentros entre distintos tipos de conocimiento.

Otro asunto relevante en ese sentido es la necesidad de entender y profundizar en la racionalidad de las políticas, los programas e instrumentos (e. g. regalías para CTeI) que fi- nancian a privados con recursos públicos para el fortalecimiento de capacidades, en donde cabe preguntarse en qué medida están estas capacidades orientadas hacia la cogestión del conocimiento local.

Específicamente, es importante volver sobre la idea de evaluación de políticas públicas de CTeI y rescatar la discusión académica acerca de la medición de la incidencia de programas que fueron diseńados para que la ciencia aportara a la construcción de paz en territorios específicos (p. ej.: el programa diseńado entre el antiguo Colciencias y el Programa de las Naciones Unidas para el Desarrollo (PNUD) para atender con conocimiento problemas presentados en regiones afectadas por la violencia).

\section{A manera de cierre}

La relación entre ciencia, producción de conocimiento y paz no es un tema visible en la agenda de estudios de paz y conflicto. Dada la coyuntura reciente del país se ha logrado algo de reconocimiento en el campo de las políticas públicas de CTeI que abogan por la inclusión social y la transformación de problemas complejos como los abordados en los Objetivos de Desarrollo Sostenible (oDs).

En la convocatoria para la realización del taller "Hacia una agenda de investigación sobre conocimiento científico, sociedad y construcción de paz en Colombia” fue sorprendente ver cómo muchas de las propuestas presentadas por académicos de universidades y centros de investigación tenían un bajo nivel de problematización sobre la relación ciencia, conocimiento y paz, que era el tema convocado. Dichas propuestas dejaban entrever una 
concepción bastante lineal de la producción del conocimiento para el estudio y la construcción de la paz. Linealidad manifiesta en la automaticidad con que asumen que la validación académica de sus trabajos, inmediatamente concede la pertinencia para entender la complejidad del tema.

Sibien producir conocimiento para entender el fenómeno de la paz e identificar las causas de la violencia necesita de la validación de las instituciones tradicionales de investigación, también es cierto que el alto componente de práctica que implica estudiar la generación de violencia y la emergencia de los conflictos obliga a girar la cabeza y revisar otras fuentes de conocimiento. Esto es viable tanto para mejorar el entendimiento del fenómeno de la paz, como para encontrar estrategias que le permitan mantenerse a lo largo del tiempo.

Los artículos presentados en este dossier nos dan pistas de que el conocimiento científico, y las otras formas de conocimiento involucradas en el estudio y la construcción de la paz, se superponen y dialogan en algunos momentos para producir un mejor entendimiento del fenómeno estudiado. Encontrar esos puntos de diálogo y esas superposiciones está dentro de las posibilidades de una agenda de investigación transformativa.

\section{REFERENCIAS}

Alger, C. F. (2007). Peace studies as a transdisciplinary project. En Handbook of Peace and Conflict Studies (pp. 299-318). London: Routledge.

Anctil Avoine, P. y Bolívar Durán, J. F. (2018). 'Toys for reconciliation': A grassroots peacebuilding initiative in Bucaramanga, Colombia. Journal of Peacebuilding \& Development, 13(1), 62-78.

Arond, E., Calvo, O., Plazas, A. y Sotelo, M. (2018). Hacia la construcción de una agenda de política de innovación transformativa para la agrocadena del café en el marco del acuerdo de paz para el departamento del Cauca. En M. Ramírez y L. Pinzón Vargas (eds.), Orientaciones para la formulación de politicas regionales de innovación transformativa en Colombia (pp. 55-57). Brighton y Bogotá: University of Sussex - Colciencias.

Asamblea General de las Naciones Unidas (1988). Resolución 43/61 del 6 diciembre de 1988, "Ciencia y paz”. Recuperado de http:// research.un.org/es/ docs/ga/quick/regular/43

Balanzó Guzmán, A. (2016). Unfolding capacity: Strategies of farmers' organizations as change agents. $(\mathrm{PhD})$, Universiteit Twente.

Balanzó, A., Nupia, C. M. y Centeno, J. P. (2019). Conocimiento investigación científica y construcción de paz. Policy Brief5, Instituto Colombo-Alemán para la Paz - CAPAZ.

Baú, V. (2015). Participatory photography for peace: Using images to open up dialogue after violence. Journal of Peacebuilding \& Development, 10(3), 74-88.

Bergmann, J. (2018). Turning an idea into reality: Creating the European Institute of Peace. Cooperation and Conflict, 53(3), 375-391.

Brynen, R. (2014) Teaching about peace operations. International Peacekeeping, 21(4), 529-538. 10.1080/13533312.2014.946740

Cajigas-Rotundo, J. C. (2007). La biocolonialidad del poder. Amazonía, biodiversidad y ecocapitalismo. El giro decolonial: reflexiones para una diversidad epistémica más allá del capitalismo global (pp. 169194). Bogotá: Pontificia Universidad Javeriana, 
IESCo-Universidad Central/Siglo del Hombre Editores.

Clubb, G. (2014). "From terrorists to peacekeepers": The ira's disengagement and the role of community networks. Studies in Conflict \& Terrorism, 37(10), 842861. 10.1080/1057610X.2014.941434

Colebatch, H. K., Hoppe, R. y Noordegraaf, M. (2010). Working for policy. Amsterdam: Amsterdam University Press.

Cummings, S., Regeer, B., de Haan, L., Zweekhorst, M. y Bunders, J. (2017). Critical discourse analysis of perspectives on knowledge and the knowledge society within the Sustainable Development Goals. Development Policy Review. 10.1111/dpr.12296

Díazgranados, S., Noonan, J., Brion-Meisels, S., Saldarriaga, L., Daza, B. C., Chávez, M. y Antonellis, I. (2014). Transformative peace education with teachers: Lessons from Juegos de Paz in rural Colombia. Journal of Peace Education, 11(2), 150 161. 10.1080/17400201.2014.898627

Dunsire, A. (1996). Tipping the balance: Autopoiesis and governance. Administration \& Society, 28(3), 299-334.

Dussel, E. (1994). 1492. El encubrimiento del otro: hacia el origen del "mito de la modernidad". La Paz: Plural Editores.

Dutrénit, G. y Sutz, J. (eds.) (2014). National innovation systems, social inclusion and development. En The Latin American Experience. Cheltenham: Edward Elgar Publishing.

Elzen, B., Geels, F. W. y Green, K. (2004). System innovation and the transition to sustainability: Theory, evidence and policy. Cheltenham: Edward Elgar Publishing.

Escobar, A. (2015). Degrowth, postdevelopment, and transitions: A preliminary conversation. Sustainability Science, 10(3), 451-462. 10.1007/ s11625-015-0297-5
Escobar, A. (2017). Autonomía y diseño: la realización de lo comunal. Buenos Aires: Tinta Limón Ediciones.

Eslami-Somea, R. y Movassagh, H. (2014). Peace education in Iran: Challenges and prospects. Journal of Peacebuilding \& Development, 9(2), 33-48.

Espinosa, M. (2007). Ese indiscreto asunto de la violencia: Modernidad, colonialidad y genocidio en Colombia. En S. Castro-Gómez y R. Grosfoguel (comps.), El giro decolonial: reflexiones para una diversidad epistémica más allá del capitalismo global (pp. 267-288). Bogotá: Siglo del Hombre Editores.

Facio Lince, L. (2019). Ciencia, Tecnología e Innovación (CTeI) y la construcción de paz en Colombia: Laboratorio de Innovación para la Paz. Documentos de Politicas Públicas, (12).

García Chueca, E. (2014). Aprender del Sur. El pensamiento de Boaventura de Sousa Santos en la transición paradigmática. Alice Working Papers, (1), 1-25.

Geels, F. W. (2010). Ontologies, socio-technical transitions (to sustainability), and the multi-level perspective. Research Policy, 39(4), 495-510.

Geels, Frank W. (2002). Technological transitions as evolutionary reconfiguration processes: A multilevel perspective and a case-study. Research Policy, 31(8-9), 1257-1274. http://dx.doi.org/10.1016/ S0048-7333(02)00062-8

Gieryn, T. F. (1983). Boundary-work and the demarcation of science from non-science: Strains and interests in professional ideologies of scientists. American Sociological Review, 48, 781-795.

Grin, J., Rotmans, J. y Schot, J. (2010). Transitions to sustainable development: new directions in the study of long term transformative change (vol. 1). London y New York: Routledge. 
Hetou, G. (2018). Middle powers' crucial peace dividend: Networking development. Journal of Peacebuilding \& Development, 13(1), 16-31.

Hidalgo, L. (2014). Ciencia, tecnología e innovación para la paz (Tesis presentada como requisito parcial para optar al título de Magíster en Estudios Políticos). Universidad Nacional de Colombia, Bogotá.

Hoppe, R. (2005). Rethinking the science-policy nexus: From knowledge utilization and science technology studies to types of boundary arrangements. Poiesis \& Praxis, 3(3), 199-215.

Hoppe, R. (2010a). From 'knowledge use' towards 'boundary work'. Sketch of an emerging new agenda for inquiry into science-policy interaction. En R. J. Veld (ed.), Knowledge democracy. Consequences for Science, Politics and Media (pp. 169-186). Heidelberg: Springer.

Hoppe, R. (2010b). Lost in translation? A boundary work perspective on making climate change governable. En P. L. P. Driessen y W. van Viersen. (eds.), From Climate Change to Social Change: Perspectives on Science-Policy Interactions (pp. 109130). Utrecht: International Books.

Ide, T. (2018). Does environmental peacemaking between states work? Insights on cooperative environmental agreements and reconciliation in international rivalries. Journal of Peace Research, 55(3), 351-365.

Jasanoff, S. (2004). States of knowledge: The co-production of science and the social order. London: Routledge.

Krampe, F. (2017). Water for peace? Post-conflict water resource management in Kosovo. Cooperation and Conflict, 52(2), 147-165.

Kuhlmann, S. y Ordóńez-Matamoros, G. (eds.). (2017). Research handbook on innovation governance for emerging economies. Towards better models. Cheltenham: Edward Elgar Publishing.
Lundgren, M. (2016). Conflict management capabilities of peace-brokering international organizations, 1945-2010: A new dataset. Conflict Management and Peace Science, 33(2), 198-223.

Marijnen, E. y Schouten, P. (2019). Electrifying the green peace? Electrification, conservation and conflict. Eastern Congo, Conflict, Security \& Development, 19(1), 1534. 10.1080/14678802.2019.1561615

Maturana, H. R. (1994). La democracia es una obra de arte (vol. 14). Bogotá: Cooperativa Editorial Magisterio.

Mignolo, W. (2002). Posoccidentalismo: las epistemologías fronterizas y el dilema de los estudios (latinoamericanos) de área (1996). Revista Iberoamericana, 68(200), 847-86. 10.5195/reviberoamer. 2002.5978

Miklian, J. y Hoelscher, K. (2018). A new research approach for peace innovation. Innovation and Development, 8(2), 189-207. 10.1080/2157930X.2017.1349580

Millar, G. (2018). Decentring the intervention experts: Ethnographic peace research and policy engagement. Cooperation and Conflict, 53(2), 259-276.

Molas-Gallart, J. (2010). Innovation, defence and security. En R. Smits, S. Kuhlmann y P. Shapira (eds.), The Theory and Practice of Innovation Policy. An International Research Handbook (pp. 247-274). Cheltenham: Edward Elgar Publishing.

Mollinga, P. (2008). The rational organisation of dissent: Boundary concepts, boundary objects and boundary settings in the interdisciplinary study of natural resources management. ZEF working paper series, (33). Recuperado de https://www. econstor.eu/handle/10419/88377

Münch, P. y Veit, A. (2018). Intermediaries of intervention: How local power brokers shape external peace- and state-building in Afghanistan and 
Congo. International Peacekeeping, 25(2), 266292. 10.1080/13533312.2017.1411808

Oksamytna, K. (2018). Policy entrepreneurship by international bureaucracies: The evolution of public information in un peacekeeping. International Peacekeeping, 25(1), 79-104. 10.1080/13533312.2017.1395286

Ordóñez-Matamoros, G., Centeno, J. P., Arond, E., Jaime, A. y Arias, K. (2018). La paz y los retos de la política de ciencia, tecnología e innovación en Colombia. En C. Soto (ed.), Seguimiento y análisis de políticas públicas en Colombia (Anuario 2017, pp. 137-168). Recuperado de https://publicaciones.uexternado.edu.co/pageflip/acceso-abierto/ pdf/anuario-seguimiento-y-analisis-2017.pdf

Ordónez-Matamoros, G., Tadlaoui, S., Porras, S., Duarte, J., López, L., Martínez, L. y CalderónPeña, G. (2013). Manual de análisis y diseño de politicas públicas. Bogotá: Universidad Externado de Colombia.

Orozco, L. A., Chavarro, D. A. y Robert, K. M. (2010). La ciencia como institución. Revista de Estudios Sociales, 37, 143-162.

Ostrom, E. (1996). Crossing the great divide: Coproduction, synergy, and development. World Development, 24(6), 1073-1087. http://dx.doi. org/10.1016/0305-750X(96)00023-X

Peñuela Herrera, J. E. (ed.) (2019). Memorias $1^{\text {er }}$ Congreso Internacional Gobernanza de la Ciencia y la Innovación. Hacia el desarrollo inclusivo. Revista Innovación y Ciencia, XXVI(2). Recuperado de https://innovacionyciencia.com/revista/101

Perera, S. (2017). To boldly know: Knowledge, peacekeeping and remote data gathering in conflict-affected states. International Peacekeeping, 24(5), 803822. 10.1080/13533312.2017.1383566

Pérez-Viramontes, G. y Restrepo-Mesa, M. (2014). Ciencia, tecnología e innovación para una Cultura de Paz. Una experiencia en el Programa OndasColciencias. Ra Ximhai, 10(2), 217-234. Recuperado de http://www.revistas.unam.mx/index. $\mathrm{php} / \mathrm{rxm} /$ article/view/65739

Reimers, B. C. (2016). Peacebuilding in refugee resettlement communities: Using photovoice to find common ground. Journal of Peacebuilding \& Development, 11(3), 108-113.

Rip, A. y Voß, J. P. (2013). Umbrella terms as mediators in the governance of emerging science and technology. Science, technology \& innovation studies: STI studies, 9(2), 39-59.

Rodríguez-Camargo, C. y Ochoa-Duarte, A. (2018). Caracterización de la Paz en Colombia como escenario de construcción de nuevas pedagogías en ciencia y tecnología. International Journal of Engineering, Social Justice and Peace, 6(1), 68-91.

Salazar, M., Lozano-Borda, M. y Lucio-Arias, D. (2014). Science, technology and innovation for inclusive development in Colombia: Pilot programmes developed by Colciencias. En G. Dutrénit y J. Sutz (eds.), National Innovation Systems, Social Inclusion and Development. The Latin American Experience (pp. 133-168). Cheltenham: Edward Elgar Publishing.

Santos, B. d. S. (2006). La sociología de las ausencias y la sociología de las emergencias: para una ecología de saberes. En Clacso (eds.), Renovar la teoría crítica y reinventar la emancipación social. Encuentros en Buenos Aires (pp. 13-41). Buenos Aires: Clacso Biblioteca Virtual.

Santos, B. d. S. (2007). Beyond abyssal thinking: From global lines to ecologies of knowledges. Review (Fernand Braudel Center), 30(1), 45-89.

Schot, J. y Geels, F. (2008). Strategic niche management and sustainable innovation journeys: Theory, findings, research agenda, and policy. Technology Analysis \& Strategic Management, 20(5), 537-554. 
Schot, J. y Steinmueller, E. (2016). Framing innovation policy for transformative change: Innovation policy 3.0. SPRU Science Policy Research Unit. Brighton: University of Sussex.

Schot, J. y Steinmueller, W. E. (2018). Three frames for innovation policy: R\&D, systems of innovation and transformative change. Research Policy, 47(9), 1554-1567.

Shehi, R. Z., Ozcan, S. y Hagen, T. (2018). The role of higher education institutions in building a culture of peace: An albanian case. Journal of Peacebuilding \& Development, 13(1), 46-61.

Spillane, J. (2015). ICT4P: Using information and communication technology for peacebuilding in Rwanda. Journal of Peacebuilding \& Development, 10(3), 97-103.

van Oost, E., Kuhlmann, S., Ordóñez-Matamoros, G. y Stegmaier, P. (2016). Futures of science with and for society: Towards transformative policy orientations. Foresight, 18(3), 276-296. 10.1108/ FS-10-2014-0063

Verbeek, J. y Osorio Rodarte, I. (2015). Increasingly, inequality within, not across, countries is rising. World Bank Blogs.

Walsh, C. (2007). Interculturalidad y colonialidad del poder. Un pensamiento y posicionamiento "otro" desde la diferencia colonial. En S. Castro-Gómez y R. Grosfoguel (comps.), El giro decolonial. Reflexiones para una diversidad epistémica más allá del capitalismo global (pp. 47-62). Bogotá: Siglo del Hombre Editores.

Yalçınkaya, H. y Özer, Y. (2017). Another lesson learned in Afghanistan: The concept of cultural intelligence. International Peacekeeping, 24(3), 434460. 10.1080/13533312.2016.1244485 